\title{
Application of improved time-frequency representation to local noise removal
}

\author{
Baotong Liu' ${ }^{1}$ - Qiyuan $\mathrm{Liu}^{2} \cdot$ Xuefu Kang ${ }^{1}$
}

Received: 28 February 2021 / Accepted: 18 April 2021 / Published online: 24 April 2021

(c) The Author(s) 2021

\begin{abstract}
The temporal resolution of conventional S transform (ST) is not sufficient for the separation of local coherent noise. We present a revised S transform (RST) which uses an analyzing window function with two control parameters of the scalar $\sigma$ and the exponential factor $\gamma$. Selecting proper parameter values (say $\sigma=1.1, \gamma=1.08$ ), the time-frequency representation (TFR) acquired by our method exhibits a higher temporal resolution. Applying an appropriate filter in the time-frequency domain, we are able to remove specific local noise. Distributed acoustic sensing (DAS) VSP section may suffer from fiber cable coupling noise, hindering the subsequent data processing and geologic interpretation. The real data example shows the coupling noise occurred in the DAS VSP can be removed by the presented RST.
\end{abstract}

Keywords The time-frequency analysis · Denoising · Distributed acoustic sensing VSP

\section{Introduction}

In seismic prospecting of petroleum and natural gas, acquired seismic data are nonstationary. Spectral decomposition transforms a seismic trace into a version of time-frequency representation (Pinnegar and Mansinha 2003; Li et al. 2016). Applying an appropriate filter in the time-frequency domain, we are able to remove specific noise (Schimmel and Gallart 2005; Parolai 2009). Researchers have proposed a variety of spectral decomposition algorithms, with each being suitable for particular applications. The $\mathrm{S}$ transform (ST) is an extension of the short-time Fourier transform (STFT) and the continuous wavelet transform (CWT) (Stockwell et al. 1996; McFadden et al. 1999). The time-frequency representation (TFR) acquired by the ST exhibits a higher temporal resolution with lower frequency resolution at high frequencies and higher frequency resolution with lower temporal resolution at low frequencies (Livanos et al. 2000; Simon et al. 2007). The low temporal resolution is disadvantageous to the separation of local coherent noise.

Baotong Liu

liubt163@163.com

1 School of Electronic Information and Electrical Engineering, Tianshui Normal University, Tianshui 741000, China

2 School of Mathematics and Statistics, Lanzhou University, Lanzhou 730000, China
For more than two decades, people have been exploring approaches to improve the spectral characteristics of the ST. As a result, various generalized S-transform (GST) arose, which allow tailoring of the analyzing windows for specific applications (McFadden et al. 1999; Pinnegar and Mansinha 2003; Li et al. 2016).

Matching pursuit gives good time-frequency resolution. However, the method is an expensive iterative technique, and sometimes it may be difficult to choose a wavelet dictionary (Wang 2007).

The Wigner-Ville distribution (WVD) is an attractive method for the spectral analysis. It can achieve very high time and frequency resolution. However, the presence of cross-term interference hinders the application of WVD. Considering that the kernel of WVD is the instantaneous autocorrelation function, Wang et al. (2020) proposed an innovative multichannel maximum-entropy method (MEM) to modify the Wigner-Ville kernel to suppress cross-term interference (Wang et al. 2020).

The W transform, recently proposed by Wang (2021), is a novel technology for the time-frequency spectral analysis. The $\mathrm{W}$ transform significantly improves the temporal resolution in TFR at low frequencies. Because the integrand is a function of $\tau$, the $\mathrm{W}$ transform cannot be implemented via a fast inverse Fourier transform (Wang 2021). 
Among methods available for time-frequency decomposition, the ST has a good compromise between effectiveness and efficiency.

Distributed acoustic sensing (DAS) is a promising tool for VSP data acquisition (Albert et al. 2013). The DAS VSP has a number of advantages over conventional VSP with geophones, such as low cost, high density, high efficiency, full vertical coverage, etc. However, DAS VSP data may suffer from fiber cable coupling noise (Willis et al. 2019). The coupling noise hinders the subsequent data processing and geologic interpretation. A few algorithms have been developed to remove the coupling noise, for instance, median filter and synthetic method (Yu et al. 2016). Different methods have their own advantages and drawbacks. Here, we try to remove the coupling noise by filtering in the time-frequency domain. The coupling noise exhibits local coherent noise with a narrow bandwidth. There are small time separation between first break (or reflection) event and the couple noise waveform. The time resolution of the ST is not high enough to meet the actual requirements. It is necessary to improve the temporal resolution in TFR.

In this paper, we present a revised S transform (RST) which uses an analyzing window function with two control parameters of the scalar $\sigma$ and the exponential factor $\gamma$. The RST is a variant of the ST, which can be viewed as a member of the GST family. The time-frequency representation (TFR) obtained by our method exhibits a higher temporal resolution. In the following sections, after describing the principle of this method, numerical experiments on two synthetic records are performed. Two synthetic experiments show that the presented RST is capable of improving the temporal resolution of TFR significantly, and we can remove the specific local signal from a given record. Then, we illustrate the effectiveness of the method with a real data example.

\section{Methodology}

The ST (Stockwell et al. 1996) is an invertible time-frequency representation method. The ST is a hybrid of STFT and CWT, containing elements of both but belonging entirely to neither category. The ST is creatively defined as (Stockwell et al. 1996; Simon et al. 2007)

$S(\tau, f)=\int_{-\infty}^{\infty} s(t) \frac{|f|}{\sqrt{2 \pi}} \exp \left[-\frac{(\tau-t)^{2} f^{2}}{2}\right] \exp [-i 2 \pi f t] \mathrm{d} t$

where $s(t)$ denotes a continuous signal being analyzed, $f$ stands for the frequency, and the quantity $\tau$ controls the position of the Gaussian window on the $t$-axis. The expression of inverse ST is given by $\mathrm{s}(t)=\int_{-\infty}^{\infty}\left[\int_{-\infty}^{+\infty} S(\tau, f) \mathrm{d} \tau\right] \exp (i 2 \pi f t) \mathrm{d} f$.

The TFR computed by the ST has lower temporal resolution at low frequencies, which is unable to meet the requirement of noise separation. Therefore, we present a revised S-transform (RST) as follows

$S(\tau, f,\{\sigma, \gamma\})=\int_{-\infty}^{\infty} s(t) \frac{\sigma|f|^{\gamma}}{\sqrt{2 \pi}} \exp \left[-\frac{(\tau-t)^{2} \sigma^{2} f^{2 \gamma}}{2}\right] \exp [-i 2 \pi f t] \mathrm{d} t$.

Parameters $\sigma$ and $\gamma$ adjust the width and waveform shape of the window with frequencies. By selecting proper values for $\sigma$ and $\gamma$, the temporal resolution of the TFR can be improved.

If energy of noise and signal become separable in the time-frequency domain, time-frequency filtering is possible. The filtered record is

$s_{\mathrm{FTR}}(t)=\int_{-\infty}^{\infty}\left[\int_{-\infty}^{+\infty} S(\tau, f) F T R(\tau, f) \mathrm{d} \tau\right] \exp (i 2 \pi f t) \mathrm{d} f$,

where $\operatorname{FTR}(\tau, f)$ is a filter.

\section{Numerical experiments with synthetic data}

Figure 1a shows a synthetic trace composed of seven Ricker wavelets with center frequencies of $[70,60,50,40,30,20$, $10] \mathrm{Hz}$. The trace length is $3.0 \mathrm{~s}$, and the sampling rate is $0.002 \mathrm{~s}$. Figure $1 \mathrm{~b}$ shows the dominant frequency estimated by the complex trace analysis, which will be used in the calculation of the $\mathrm{W}$ transform. Figure 1c shows the time-frequency spectrum computed by the $\mathrm{S}$ transform (ST). The time-frequency representation (TFR) estimated by the W transform is shown in Fig. 1d. The distribution centroid of the spectral energy corresponds to the center frequency of a wavelet. The TFR estimated by the RST is shown in Fig. 1e. Compared with Fig. 1c, the temporal resolution of both Fig. 1d, e is significantly improved.

Figure 2a shows a synthetic record consisting of a $20 \mathrm{~Hz}$ cosine wave and two Ricker wavelets for the first half, an $80 \mathrm{~Hz}$ cosine wave for the second half. The recording length is $0.4 \mathrm{~s}$, and the sampling rate is $0.0004 \mathrm{~s}$. The temporal separation between two Ricker wavelets is very small. The peak frequency of the first wavelet is $80 \mathrm{~Hz}$, and that of the latter is $90 \mathrm{~Hz}$. The ST of record of Fig. 1a is given in Fig. 2b. Because the resolution is not high enough in the time-frequency domain, it is difficult to separate the two wavelets. Figure $2 \mathrm{c}$ shows the time-frequency representation of Fig. 2a obtained by the RST Eq. $3(\sigma=1.1, \gamma=1.08)$. Values of $\sigma$ and $\gamma$ are determined by several tests. The temporal resolution is obviously improved and the wavelets energy is more concentrated. We design a time-frequency filter: 
Fig. 1 Time-frequency spectra of a synthetic trace. a A synthetic record composed of seven Ricker wavelets with center frequencies of [70, 60, 50, 40, 30, 20, 10] Hz. b Dominant frequency estimated through the complex trace analysis. c Time-frequency spectrum computed by the $\mathrm{S}$ transform (ST). d Time-frequency spectrum computed by the $\mathrm{W}$ transform (WT). e Time-frequency spectrum computed by revised $\mathrm{S}$ transform (RST) (a)

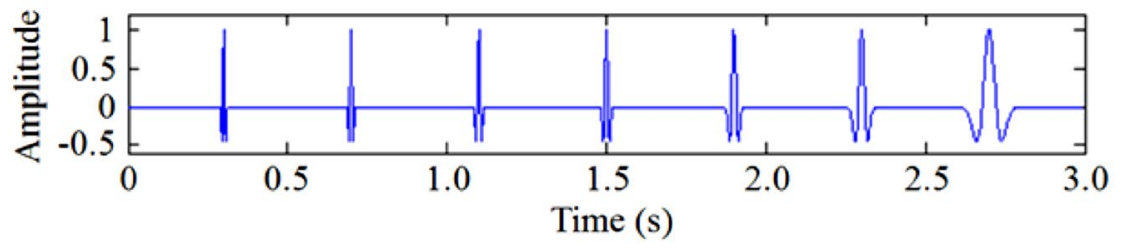

(b)

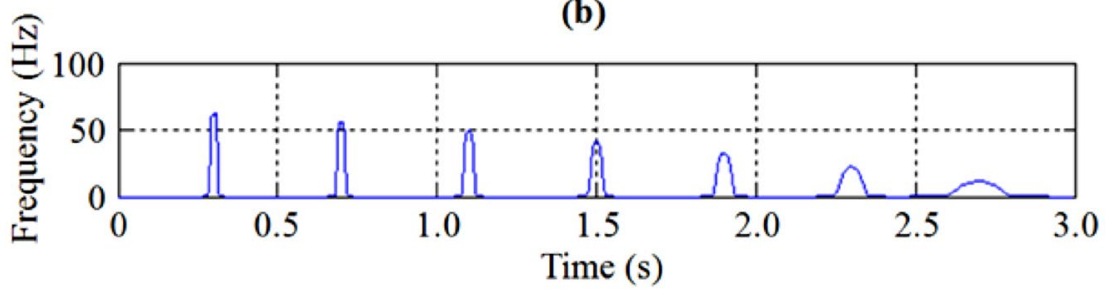

(c)

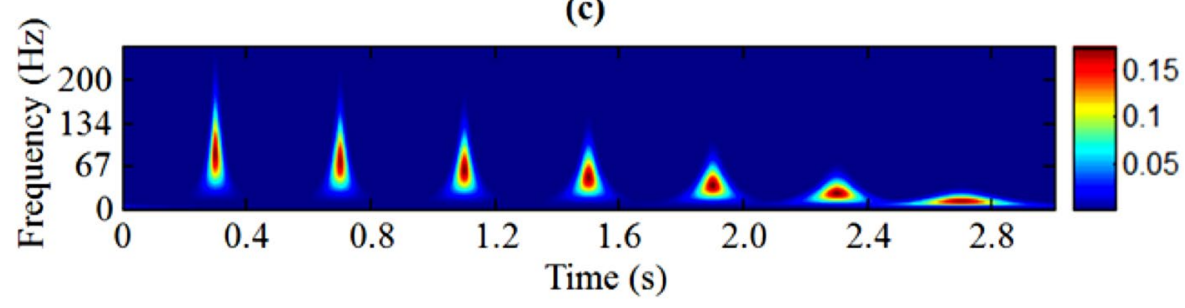

(d)

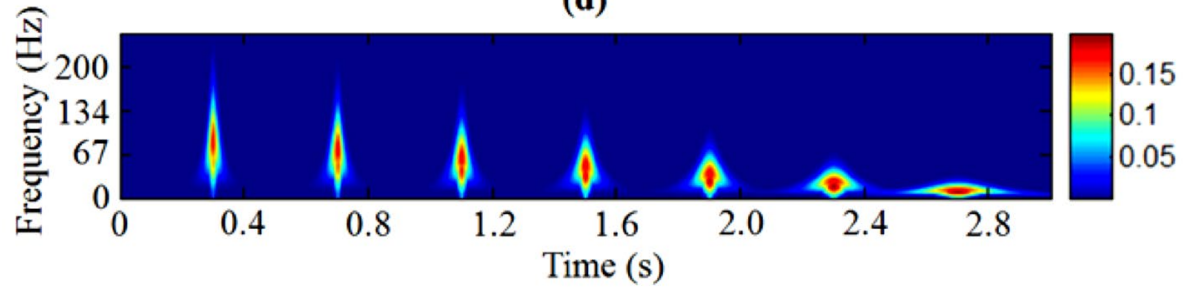

(e)

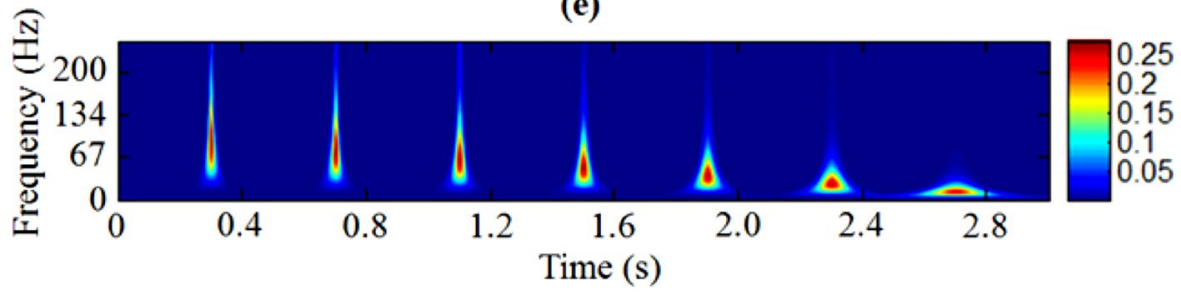

$\operatorname{FTR}(\tau, f)=\left\{\begin{array}{l}0, \\ 1,(\tau, f) \in \Omega \\ 1,(\tau, f) \notin \Omega\end{array}\right.$,

here $\Omega$ is the distribution region of energy of $90 \mathrm{~Hz}$ Ricker wavelet. Applying this filter to Fig. $2 \mathrm{c}$ gives $2 \mathrm{~d}$. The inverse S-transform of Fig. $2 d$ is shown in 2e. The difference between Fig. 2a and 2e, removed wavelet, is plotted in Fig. 2f. The result of numerical experiment illustrates that, using improved time-frequency spectrum, we can separate and remove specific noise located in a local time range.

\section{Real data example}

The DAS VSP test was carried out in an oil field in northwestern China. The field data were acquired in a vertical well. The total number of record traces is 115 . The recording length is $2.5 \mathrm{~s}$ with a sampling interval of $0.001 \mathrm{~s}$. Figure 3 shows the input data. Because of the poor coupling of the fiber-optic cable to the wellbore, local coherent noise appeared in traces 3-25, 67-78, and 84-91. We 
(a)

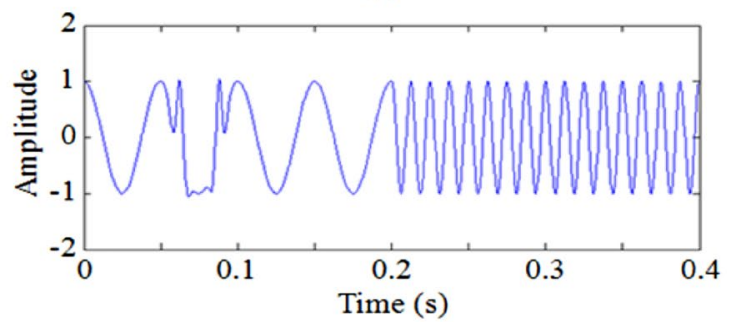

(c)

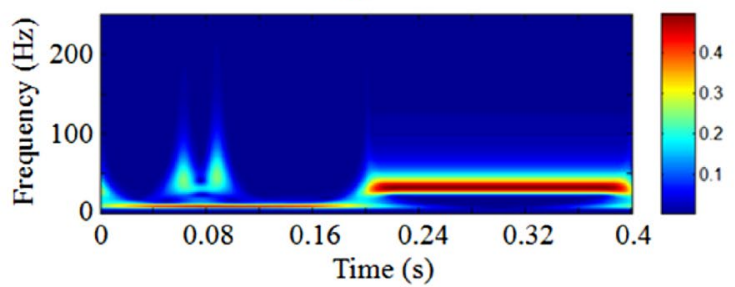

(e)

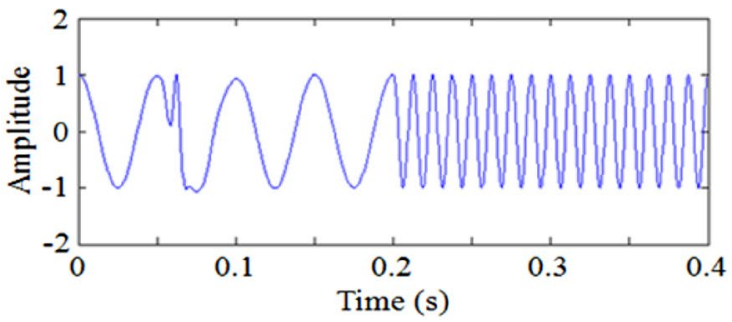

Fig. 2 a A synthetic record. The expression of vibration signal is $\mathrm{h}(t)=\cos (2 \pi * 20 * t) t \in[0,0.2], \mathrm{h}(t)=\cos (2 \pi * 80 * t) t \in(0.2,0.4]$. Peak frequencies of two Ricker wavelets are $80 \mathrm{~Hz}$ and $90 \mathrm{~Hz}$, respec-

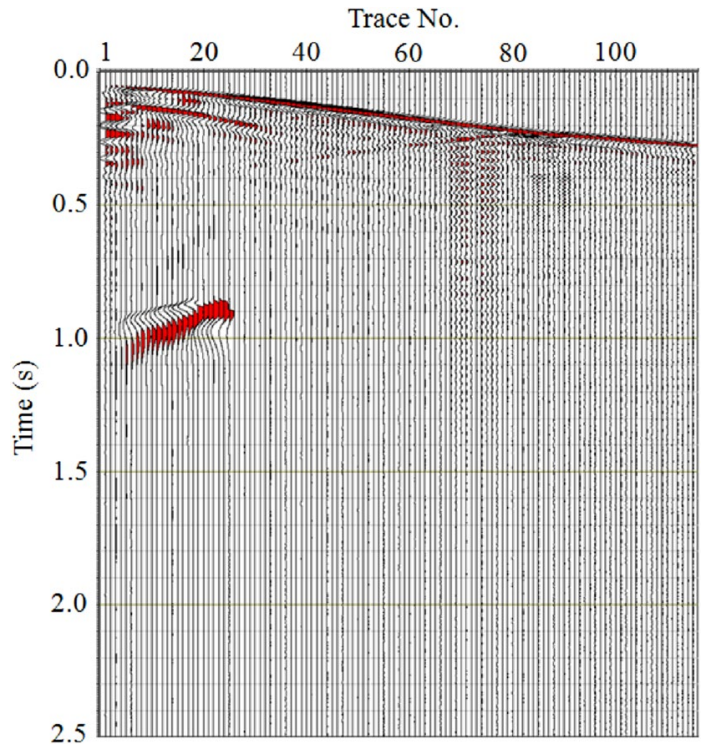

Fig. 3 Distributed acoustic sensing (DAS) VSP field data. The coupling noise appeared in traces 3-25, 67-78, and 84-91 (b)

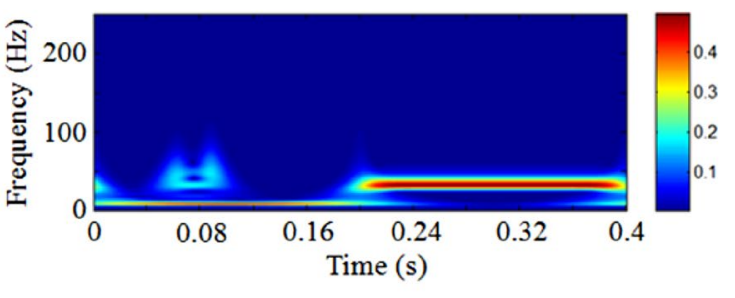

(d)

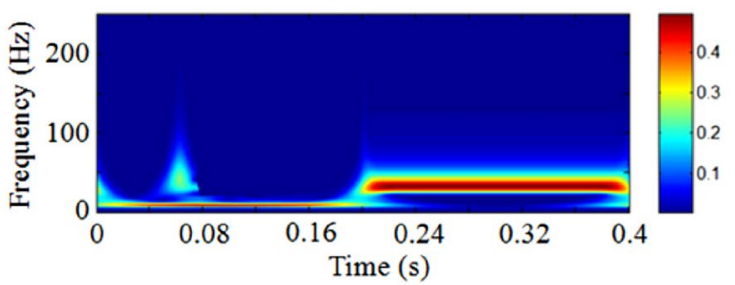

(f)

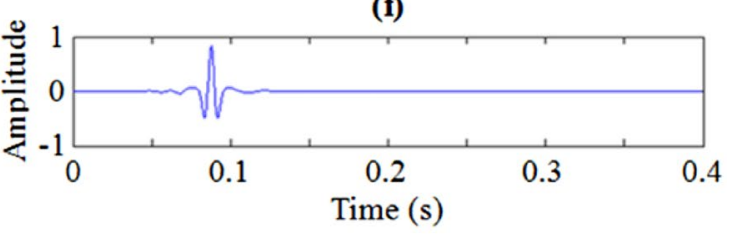

tively. b ST of a. c RST of a. d Filtered time-frequency spectrum. e Inverse ST of $\mathbf{d}$. f Removed wavelet, difference between a and $\mathbf{e}$

use the presented RST to remove the local coherent noise by filtering in the time-frequency domain. For example, Fig. 4a shows the seismogram of trace 70. The TFR of trace 70 is given in Fig. 4b. Applying a filter defined by Eq. 5, the filtered time-frequency spectrum is shown in Fig. 4c. The inverse ST of Fig. 4c gives the denoised seismogram (Fig. 4d). The removed coupling noise, the difference between Fig. 4a and Fig. 4d, is plotted in Fig. 4e. Other traces are processed in the similar way. Figure 5 shows the denoised DAS VSP data. It can be seen that the local coherent noise has been removed.

\section{Conclusions}

The time resolution of conventional ST is too low to separate the local coherent noise. We present a revised S-transform (RST) which uses an analyzing window function with two control parameters. Values of the scalar $\sigma$ and the exponential factor $\gamma$ are determined by several tests. When $\sigma=1.1$, $\gamma=1.08$, we can get a satisfactory result. The time-frequency 
Fig. 4 a Seismogram of the 70th trace in Fig. 3, contains the DAS coupling noise. b Timefrequency representation of $\mathbf{a}$. c Filtered time-frequency spectrum. d Noise removal result. $\mathbf{e}$ Removed coupling noise, difference between $\mathbf{a}$ and $\mathbf{d}$ (a)

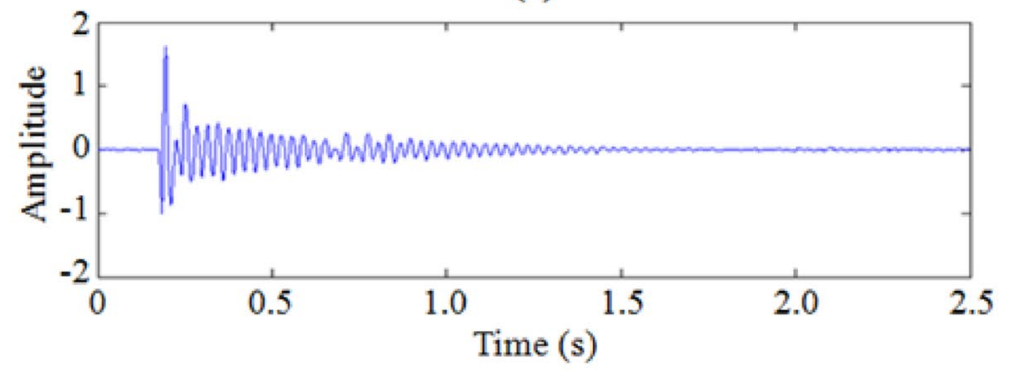

(b)

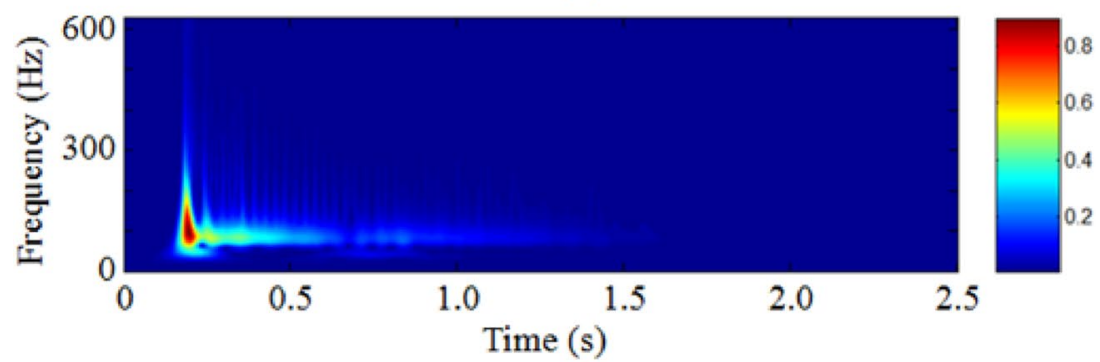

(c)

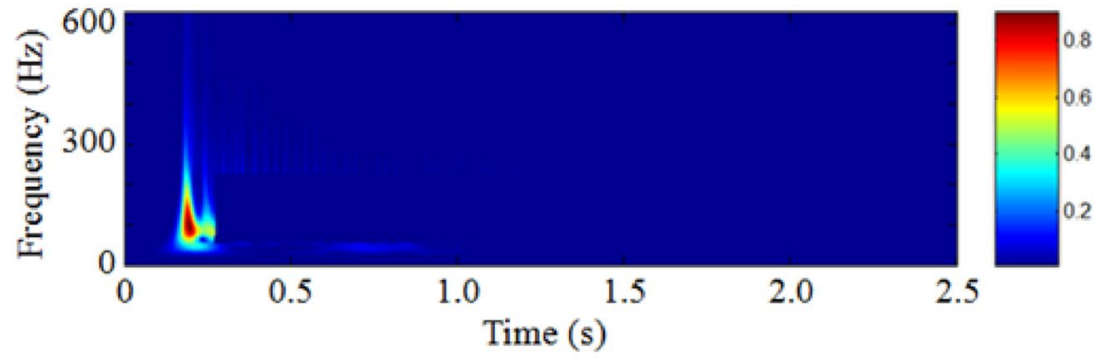

(d)

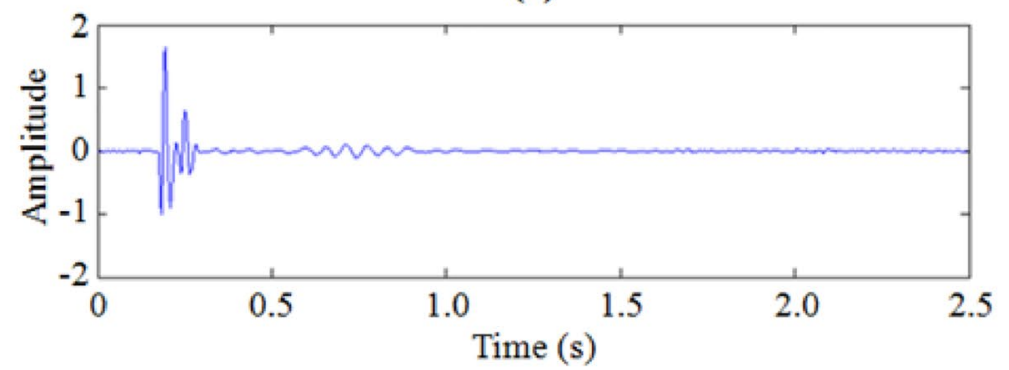

(e)

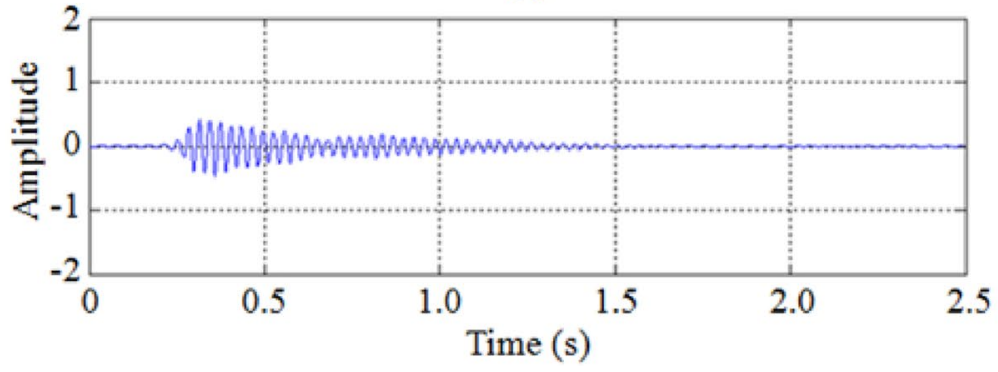




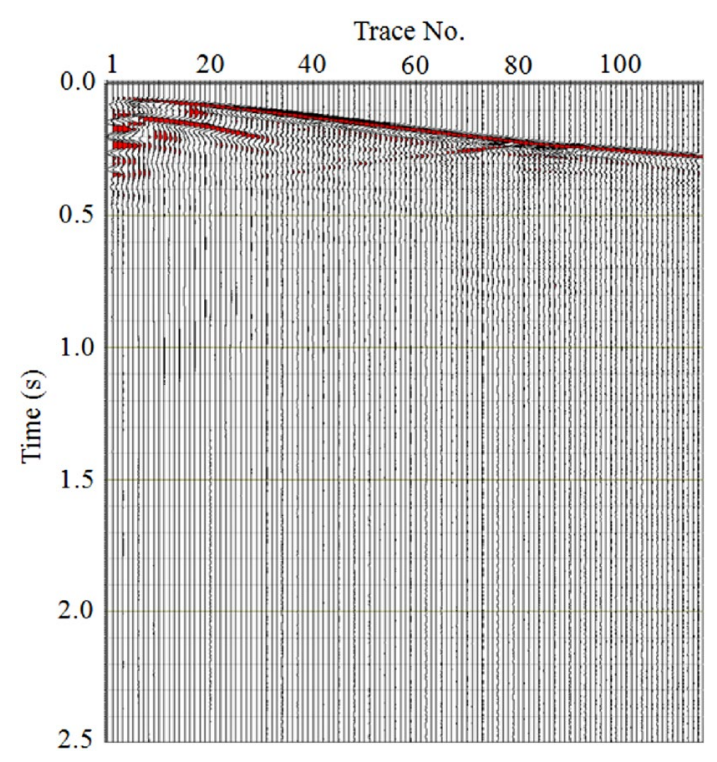

Fig. 5 Denoised DAS VSP data

representation (TFR) acquired by our method exhibits a higher temporal resolution. Applying an appropriate filter in the time-frequency domain, we are able to remove specific local noise. DAS VSP data may suffer from fiber cable coupling noise. The real data example shows the coupling noise occurred in the DAS VSP can be removed by the presented RST.

Acknowledgements The authors would like to thank the National Natural Science Foundation of China (41264002). We also thank two anonymous reviewers for their constructive comments and suggestions.

Funding This research was funded by the National Natural Science Foundation of China (Grant No. 41264002).

\section{Declarations}

Conflict of interest On behalf of all the co-authors, the corresponding author declares that there is no conflict of interest.

Open Access This article is licensed under a Creative Commons Attribution 4.0 International License, which permits use, sharing, adaptation, distribution and reproduction in any medium or format, as long as you give appropriate credit to the original author(s) and the source, provide a link to the Creative Commons licence, and indicate if changes were made. The images or other third party material in this article are included in the article's Creative Commons licence, unless indicated otherwise in a credit line to the material. If material is not included in the article's Creative Commons licence and your intended use is not permitted by statutory regulation or exceeds the permitted use, you will need to obtain permission directly from the copyright holder. To view a copy of this licence, visit http://creativecommons.org/licenses/by/4.0/.

\section{References}

Albert M, Jorge L, Jeff M et al (2013) Distributed acoustic sensing for reservoir monitoring with VSP. Lead Edge 32:1278-1283

Li D, Castagna J, Goloshubin G (2016) Investigation of generalized S-transform analysis windows for time-frequency analysis of seismic reflection data. Geophysics 81:V235-V247

Livanos G, Ranganathan N, Jang J (2000) Heart sound analysis using the S-transform. Comput Cardiol 27:587-590

McFadden PD, Cook JG, Forster LM (1999) Decomposition of gear vibration signals by the generalized S transform. Mech Syst Signal Process 13:691-707

Parolai S (2009) Denoising of seismograms using the S transform. Bull Seismol Soc Am 99:226-234

Pinnegar CR, Mansinha L (2003) The S-transform with windows of arbitrary and varying shape. Geophysics 68:381-385

Schimmel M, Gallart J (2005) The inverse S-transform in filters with time-frequency localization. IEEE Trans Signal Process 53:4417-4422

Simon C, Ventosa S, Schimmel M et al (2007) The S-transform and its inverses: side effects of discretizing and filtering. IEEE Trans Signal Process 55:4928-4937

Stockwell RG, Mansinha L, Lowe RP (1996) Localization of the complex spectrum: the $\mathrm{S}$ transform. IEEE Trans Signal Process 44:998-1001

Wang Y (2007) Seismic time-frequency spectral decomposition by matching pursuit. Geophysics 72:V13-V20

Wang Y, Rao Y, Xu D (2020) Multichannel maximum-entropy method for the Wigner-Ville distribution. Geophysics 85:V25-V31

Wang Y (2021) The W transform. Geophysics 86:V31-V39

Willis ME, Wu X, Palacios W et al (2019) Understanding cable coupling artifacts in wireline-deployed DAS VSP data. SEG 89th annual international meeting expanded abstracts, pp 5310-5314

Yu G, Cai Z, Chen Y et al (2016) Walkaway VSP using multimode optical fibers in a hybrid wireline. Lead Edge 35:615-619

Publisher's Note Springer Nature remains neutral with regard to jurisdictional claims in published maps and institutional affiliations. 